\title{
Correlates of Early Prenatal Care Access among U.S. Women: Data from the Pregnancy Risk Assessment Monitoring System (PRAMS)
}

\author{
Rebecca A. Krukowski ${ }^{1}$ - Lisette T. Jacobson ${ }^{2}$. Jemima John ${ }^{3}$. Patricia Kinser ${ }^{4} \cdot$ Kendra Campbell $^{5}$. \\ Tracey Ledoux ${ }^{6} \cdot$ Kara L. Gavin ${ }^{7} \cdot$ Chi-Yang Chiu ${ }^{1} \cdot$ Jiajang Wang ${ }^{1} \cdot$ Abbey Kruper $^{8}$
}

Accepted: 24 September 2021 / Published online: 4 October 2021

(c) The Author(s), under exclusive licence to Springer Science+Business Media, LLC, part of Springer Nature 2021

\begin{abstract}
Objectives Early first trimester prenatal counseling could reduce adverse maternal and child health outcomes. Existing literature does not identify the length of time between suspecting pregnancy and attending their first prenatal visit. Identifying this potential window for change is critical for clinical practice, intervention programming and policy change.

Methods The study sample was composed of women in the United States who responded to the Pregnancy Risk Assessment Monitoring Systems survey in 2016, for the following questions - when they first suspected pregnancy, when they attended their first prenatal visit, were they able to receive prenatal care as early as they wished, and perceived barriers to receiving prenatal care.

Results On average, participants became certain they were pregnant at $6.0(\mathrm{SE}=0.1)$ weeks gestation, while participants reported having their first prenatal care visit at $9.3(\mathrm{SE}=0.1)$ weeks, with clear health disparities by race, age, WIC participation, education level, and marital status. About $15 \%$ of women reported not receiving prenatal care as early as they wished. Structural or financial barriers in the health care system were common: $38.1 \%$ reported that no appointments available, $28.2 \%$ reported not having money or insurance to pay for the visit, $27.3 \%$ reported that the doctor or health plan would not start care, and $22.5 \%$ reported not having a Medicaid card.

Conclusions for Practice This study illustrates a window for opportunity to provide earlier prenatal care, which would facilitate earlier implementation of prenatal counseling. Strategies to address barriers to care on the patient, provider and systemic levels, particularly among vulnerable population groups, are warranted.

What is already known on this subject? Seeking prenatal care early is associated with better health outcomes for women and infants. A window of opportunity exists between suspecting pregnancy and attending a first prenatal visit.

What this study adds? Clear health disparities were apparent in both recognizing their pregnancies, and receiving early prenatal care by race, age, WIC participation, education level, and marital status. About $15 \%$ of women reported not receiving prenatal care as early as they wished, and many attributed this later care to structural or financial barriers in the health care system.
\end{abstract}

Keywords PRAMS $\cdot$ Pregnancy $\cdot$ Prenatal care $\cdot$ Barriers $\cdot$ Sociodemographic characteristics

Rebecca A. Krukowski

rkrukows@uthsc.edu

1 University of Tennessee Health Science Center, $66 \mathrm{~N}$. Pauline St., Room 316, Memphis, TN 38105, USA

2 Department of Population Health, University of Kansas, Lawrence, KS, USA

3 MD Anderson, Houston, TX, USA

4 School of Nursing, Virginia Commonwealth University, Richmond, VA, USA
Department of Psychology, University of Alaska-Fairbanks, Fairbanks, AK, USA

6 University of Houston, Houston, TX, USA

7 Department of Surgery, University of Wisconsin, Madison, WI, USA

8 Department of Obstetrics and Gynecology, Medical College of Wisconsin, Milwaukee, WI, USA 


\section{Introduction}

In the United States, the number of reported maternal pregnancy-related deaths has increased from 7.2 deaths per 100,000 live births in 1987 to 18.0 deaths per 100,000 live births in 2014 (Centers for Disease Control \& Prevention, $2019 b$ ). This increase may be due in part to increased identification and reporting of maternal deaths, and it may also be due to an increasing number of pregnant women in the U.S. who suffer from chronic health conditions, including hypertension (Kuklina et al., 2009), diabetes (Albrecht et al., 2010), and chronic heart disease (Kuklina \& Callaghan, 2011), which increase the likelihood of pregnancy complications and maternal morbidity. Moreover, significant racial and ethnic disparities in pregnancy-related mortality exist with 40.0 deaths per 100,000 live births for Black women and 17.8 deaths per 100,000 live births for women of other races/ethnicities compared to 12.4 deaths per 100,000 live births for white women (Centers for Disease Control \& Prevention, 2019b). Prenatal care is essential to optimize women's and infants' health outcomes (Academy \& of Pediatrics Committee on Fetus \& Newborn, 2012). Early prenatal care facilitates appropriate screening for medical conditions and fetal anomalies (American Academy of Pediatrics Committee on Fetus and Newborn, 2012); timely provision of gestational weight gain guidelines (Goldstein et al., 2017); and receipt of education and counseling services on health enhancing behaviors (e.g. getting adequate exercise, eating a balanced diet, use of prenatal vitamins, quitting smoking and eliminating alcohol consumption (Moos et al., 2008).

Early prenatal care presents a critical window for changes to personal and systemic factors that may compromise the health of women and infants. Despite evidence of the positive impact of these health enhancing behaviors on favorable maternal and child health outcomes and about $77 \%$ of women initiating prenatal care in the first trimester (Michelle \& Osterman, 2016), notable disparities in prenatal care access persist among U.S. women, particularly among younger, less educated, geographically isolated, and racial/ethnic-minority maternal populations (Michelle \& Osterman, 2016). Furthermore, although it is well-established that seeking early prenatal care is associated with better health outcomes for women and infants (Stulberg et al., 2017; Yan, 2017), existing literature does not identify the specific time point in pregnancy at which women typically attend their first prenatal visit or the length of time between suspecting pregnancy and attending their first prenatal visit (i.e. the potential window for change). Understanding typical timing along with barriers to accessing early prenatal care is vital for implementing best practice recommendations as well as potential systemic changes aimed at improving health outcomes.
Thus, our study aimed to address these gaps in the literature by determining when women in the 2016 Pregnancy Risk Assessment Monitoring Systems dataset first suspected pregnancy, when they attended their first prenatal visit, and their perceptions around prenatal care initiation (i.e. were they able to receive prenatal care as early as they wished?). Additionally, we examined the prevalence of perceived barriers (e.g. health care system, finances, scheduling, childcare) to receiving prenatal care as early as they wished. In doing so, our study can inform prenatal care policies and clinical practice in racially, ethnically and socioeconomically diverse populations.

\section{Methods}

Data from the 2016 PRAMS state-level datasets were aggregated prior to analysis. The goal of PRAMS national surveillance project is to monitor maternal health, identify at-risk populations, and understand the impact of health attitudes, behaviors and experiences in order to better inform and improve maternal and infant care (Centers for Disease Control \& Prevention, 2019a). Using stratified sampling, women who delivered a live baby in the previous two to four months are randomly selected from state birth certificate records for participation in PRAMS. Selected individuals are initially contacted via a mailed survey, and by phone if there is no initial response, and all participants received a written informed consent statement. Standardized data collection procedures and measures allow comparisons among states. All states administered the core questions, and a subset of states administered each standard question. For a thorough description of PRAMS study methods, see (Shulman et al., 2006).

\section{Measures}

Maternal characteristics obtained from the birth certificate data included ethnicity, race, marital status, educational attainment, maternal age, and participation in the Nutrition Program for Women, Infants, and Children (WIC). All other variables were collected from the PRAMS questionnaire data. Using self-reported anthropometric data (i.e. height, pre-pregnancy weight), a pre-pregnancy body mass index (BMI) variable was created using the standard formula for BMI. A categorical BMI variable was then created using the National Institutes of Health, Heart, Lung, and Blood Institute BMI categories of underweight $(<18.5 \mathrm{~kg} /$ $\mathrm{m}^{2}$ ), normal $\left(18.5-24.9 \mathrm{~kg} / \mathrm{m}^{2}\right)$, overweight (25.0-29.9 kg/ $\left.\mathrm{m}^{2}\right)$ and obese $\left(\geq 30.0 \mathrm{~kg} / \mathrm{m}^{2}\right)$.

Gestational age at first prenatal care visit was measured by the core question, "How many weeks or months pregnant were you when you had your first visit for prenatal 
care?" with data available from 26 states (i.e. Alaska, Arkansas, Colorado, Delaware, Iowa, Illinois, Louisiana, Massachusetts, Maryland, Maine, Michigan, Missouri, New Hampshire, New Jersey, New Mexico, New York, Oklahoma, Pennsylvania, Rhode Island, Texas, Utah, Vermont, Washington, Wisconsin, West Virginia, and Wyoming) and New York City. Gestational age at pregnancy confirmation was measured by the standard question, "How many weeks or months pregnant were you when you were sure you were pregnant? For example, you had a pregnancy test or a doctor, nurse or other health worker said you were pregnant" with data available from four states (i.e. Delaware, Maine, New Jersey and Oklahoma). Satisfaction with timing of first prenatal care visit was measured by the standard question, "Did you get prenatal care as early in your pregnancy as you wanted?" with data available from 12 states (i.e. Alaska, Delaware, Illinois, Louisiana, Maine, Michigan, Missouri, New Jersey, New Mexico, Pennsylvania, Texas, and Wisconsin). Barriers to receiving early prenatal care were assessed via "yes" or "no" responses to the following prompts: "Did any of these things keep you from getting prenatal care when you wanted it? 1) I couldn't get an appointment when I wanted one; 2) I didn't have enough money or insurance to pay for my visits; 3) I didn't have any transportation to get to the clinic or doctor's office; 4) The doctor or my health plan would not start care as early as I wanted; 5) I had too many other things going on; 6) I couldn't take time off from work or school; 7) I didn't have my Medicaid card; 8) I didn't have anyone to take care of my children; 9) I didn't know that I was pregnant; 10) I didn't want anyone else to know I was pregnant; and 11) I didn't want prenatal care." Multiple items could be selected. Data were available for this question from 11 states (i.e. Arkansas, Delaware, Illinois, Louisiana, Michigan, Missouri, New Jersey, New Mexico, Pennsylvania, Texas, and Wisconsin).

\section{Sample}

The study sample was composed of women who responded to the PRAMS survey in 2016, for each question that had data available for release. PRAMS had a 55\% minimum overall response rate threshold policy for the release of 2016 data. The weighted response rate for the included states ranged from $54.6 \%$ to $72.5 \%$. The study was determined to be exempt from University of Tennessee Health Science Center Institutional Review Board approval because no identifiable private information was utilized and have therefore been performed in accordance with the ethical standards laid down in the 1964 Declaration of Helsinki and its later amendments.

\section{Data Analysis and Statistical Methods}

Data were analyzed using SAS version 9.4 with survey procedures. In univariate analyses, differences of marginal means and 95\% Confidence Intervals (CIs) were generated through one-way ANOVA models; odds ratios and 95\% CIs were generated through simple logistic regression. Multiple linear and logistic regression models were used to determine significant factors of continuous and binary outcomes, respectively. Missing data were handled using pairwise deletion. All tests were 2 -sided, and significance was set at $P<0.05$.

\section{Results}

The sample included 31,642 women. Most women were 18-34 years of age, were non-Hispanic white, were married, had at least one previous live birth, and had a high school degree or higher (Table 1).

\section{Gestational Age When Certain of Pregnancy}

Participants $(n=4677)$ from four states (i.e. Delaware, Maine, New Jersey, and Oklahoma) responded to when they were certain they were pregnant. Eighty-five percent of respondents were certain they were pregnant at 8 weeks gestation or before (Table 2). On average, participants became certain they were pregnant at $6.0(\mathrm{SE}=0.1)$, median $=4.5(\mathrm{SE}=0.1)$ weeks gestation. Significant differences were detected in gestation weeks when certain of pregnancy by race, ethnicity, age, WIC participation, education level, number of previous births and marital status (Table 3). Women who identified as white became aware of their pregnancies significantly earlier $($ mean $(\mathrm{SE})=5.7(0.1)$ weeks $)$ than women who identified as Black and Native North American (mean $(\mathrm{SE})=7.2(0.3)$ weeks and mean $(\mathrm{SE})=7.0(0.3)$ weeks, respectively). Women who identified as Hispanic reported pregnancy certainty significantly later $(\operatorname{mean}(\mathrm{SE})=6.6(0.2)$ weeks $)$ compared to women who did not identify as Hispanic $(\operatorname{mean}(\mathrm{SE})=5.9(0.1)$ weeks $)$. Women who were receiving WIC benefits were certain of pregnancy significantly later (mean $(\mathrm{SE})=6.6(0.1)$ weeks) than women who were not $($ mean $(\mathrm{SE})=5.7(0.1)$ weeks). Younger women (i.e. 24 years or younger) were certain of their pregnancy significantly later than women who were 35 years or older. In addition, women who reported education levels less than a bachelor's degree were certain of their pregnancy significantly later than women with a bachelor's degree. Women who had no previous births or 1-2 previous births were certain of their pregnancy earlier than women with 
Table 1 Socio-demographic Characteristics

\begin{tabular}{|c|c|}
\hline Characteristic & $\begin{array}{l}\text { Mean (Standard } \\
\text { Deviation) or } \mathrm{N}^{\mathrm{a}} \\
(\%)^{\mathrm{b}}\end{array}$ \\
\hline \multicolumn{2}{|l|}{ Race } \\
\hline White & $18,616(71.1 \%)$ \\
\hline Black & $5565(13.5 \%)$ \\
\hline Asian (Chinese, Japanese, Filipino, other Asian) & $1941(6.0 \%)$ \\
\hline Native North American (American Indian, Hawaiian, Alaskan native) & $1219(0.9 \%)$ \\
\hline Other races & $1685(5.9 \%)$ \\
\hline Mixed race & $1310(2.6 \%)$ \\
\hline Hispanic & $6193(23.9 \%)$ \\
\hline \multicolumn{2}{|l|}{ Age (years) } \\
\hline$<18$ & $456(1.5 \%)$ \\
\hline $18-24$ & $7335(22.4 \%)$ \\
\hline $25-34$ & $18,232(58.6 \%)$ \\
\hline$\geq 35$ & $5619(17.4 \%)$ \\
\hline \multicolumn{2}{|l|}{ Nutrition program for Women, Infant and Child participation } \\
\hline No & $18,507(61.3 \%)$ \\
\hline Yes & $12,619(38.7 \%)$ \\
\hline \multicolumn{2}{|l|}{ Maternal years of education } \\
\hline$<8$ th grade & $996(3.4 \%)$ \\
\hline $9-12$ th grade & $3313(10.3 \%)$ \\
\hline High school graduate/GED & $7744(24.4 \%)$ \\
\hline Some college/Associate degree & $8725(26.3 \%)$ \\
\hline Bachelor's degree or higher & $10,448(35.6 \%)$ \\
\hline \multicolumn{2}{|l|}{ Number of previous live births } \\
\hline 0 & $12,428(38.2 \%)$ \\
\hline $1-2$ & $15,155(50.2 \%)$ \\
\hline$\geq 3$ & $3991(11.6 \%)$ \\
\hline Married & $18,942(61.9 \%)$ \\
\hline Pre-pregnancy BMI $\left(\mathrm{kg} / \mathrm{m}^{2}\right)$ & $26.7(0.06)$ \\
\hline \multicolumn{2}{|l|}{ Pregnancy BMI category } \\
\hline Underweight $(<18.5)$ & $1113(3.6 \%)$ \\
\hline Normal (18.5-24.9) & $14,113(47.7 \%)$ \\
\hline Overweight (25-29.9) & $7503(25.5 \%)$ \\
\hline Obese $(\geq 30)$ & $7471(23.2 \%)$ \\
\hline
\end{tabular}

${ }^{a}$ Number of women from unweighted sample distribution

${ }^{\mathrm{b}}$ Percentages were weighted to account for deliberate survey oversampling and nonresponse and non-coverage

BMI Body Mass Index

3 or more previous births. Women who were not married were certain of their pregnancy significantly later (mean $(\mathrm{SE})=7.1(0.2)$ weeks) than women who were married (mean $(\mathrm{SE})=5.4(0.1)$ weeks). However, no significant differences were detected in timing of pregnancy certainty by BMI category. In a multiple linear regression model (including all covariates), characteristics significantly associated with the timing of being certain of pregnancy were race, age, education, number of previous births, marital status, and BMI category (Table 4).

\section{Gestation Age at First Prenatal Care Visit}

Sixty-four percent of respondents received prenatal care prior to 8 weeks gestation, and $28 \%$ of women received prenatal care between 9 and 13 weeks gestation (Table 2). On average, participants reported having their first prenatal care visit at 9.3 $(\mathrm{SE}=0.1$, median $=7.3(\mathrm{SE}=0.0)$ weeks gestation (data from 26 states and New York City, $n=30,087)$. With a similar pattern of results to the certainty of pregnancy data, significant differences were shown in gestation weeks at which prenatal care was received by race, age, WIC participation, education level, 
Table 2 The number of weeks pregnant when participants were certain they were pregnant and when they had their first prenatal visit with a healthcare provider

\begin{tabular}{ll}
\hline Characteristic & $\mathrm{N}^{\mathrm{a}}(\%)^{\mathrm{b}}$ \\
\hline $\begin{array}{l}\text { Gestational } \\
\text { age when }\end{array}$ & \\
certain of \\
pregnancy \\
$\leq 8$ weeks & $3902(85.1 \%)$ \\
$9-13$ weeks & $569(11.2 \%)$ \\
$14-27$ weeks & $174(2.8 \%)$ \\
$\geq 28$ weeks & $32(0.9 \%)$ \\
Gestational & \\
age at 1 st \\
prenatal visit \\
$\leq 8$ weeks & $19,486(64.1 \%)$ \\
$9-13$ weeks & $8724(28.4 \%)$ \\
$14-27$ weeks & $2159(6.9 \%)$ \\
$\geq 28$ weeks & $218(0.7 \%)$ \\
\hline a Number of $\quad$ women from \\
unweighted sample distribution \\
b Percentages were weighted to \\
account for deliberate survey \\
oversampling and nonresponse \\
and non-coverage
\end{tabular}

number of previous births and marital status (Table 3). Women who identified as white received prenatal care significantly earlier $($ mean $(\mathrm{SE})=8.9(0.1)$ weeks $)$ than women who identified as Black and Native North American (mean $(\mathrm{SE})=10.1(0.4)$ weeks and mean $(\mathrm{SE})=10.1(0.4)$ weeks, respectively). Women who were participating in WIC received prenatal care significantly later $($ mean $(\mathrm{SE})=9.9(0.2)$ weeks) than women who were not $($ mean $(\mathrm{SE})=8.8(0.1)$ weeks $)$. Women who were 24 years or younger received prenatal care significantly later than women who were 35 years or older. In addition, women who reported education levels less than a bachelor's degree received prenatal care significantly later than women with a bachelor's degree. Women who had 3 or more previous births received prenatal care significantly earlier than women who had no previous births or 1-2 previous births. Women who were not married received prenatal care significantly later $(\operatorname{mean}(\mathrm{SE})=10.6(0.2)$ weeks) than women who were married $(\operatorname{mean}(\mathrm{SE})=8.4(0.1)$ weeks). Finally, no significant differences were detected in timing of prenatal care by ethnicity or BMI category. In a multiple linear regression model (including all covariates), characteristics significantly associated with gestational age at first prenatal care visit were race, age, WIC participation, education, number of previous births, and marital status (Table 4).

\section{Predictors of Not Getting Prenatal Care as Early as Desired}

Participants $(n=14,330)$ in 12 states comprised the sample responding to whether they received prenatal care as early as they wished. While $84.8 \%(n=12,164)$ of women received prenatal care as early in their pregnancy as they wanted, $15.2 \%$ of women $(n=2166)$ did not. Women who received prenatal care as early as they wanted reported they received care at mean $(\mathrm{SE})=7.7(0.1)$ weeks, after being sure they were pregnant at mean $(\mathrm{SE})=5.4(0.1)$ weeks. In comparison, women who did not receive prenatal care as early as they wanted reported they received care at mean $(\mathrm{SE})=12.8(0.2)$ weeks, after being sure they were pregnant at mean $(\mathrm{SE})=8.3(0.4)$ weeks. Clear differences across race, ethnicity, age, education, WIC participation, number of previous births, marital status, and weight status were observed (Table 5). Specifically, in the univariate analyses, Black women, Native North American, women of other races, Hispanic women, women younger than 24 years old, women participating in WIC, women with less than a bachelor's degree, women with 1-2 previous births, women who were unmarried, and women with underweight were significantly more likely to report not receiving prenatal care as early as they wanted. In a multiple logistic regression model (including all covariates), characteristics associated with reporting not getting prenatal care as early as desired were ethnicity, age, education, number of previous births, and marital status (Table 6).

\section{Reasons for Not Receiving Early Prenatal Care}

Participants in 11 states comprised the sample responding to barriers to receiving early prenatal care. Of the women who did not receive early prenatal care, $42.3 \%$ reported the reason was not being aware they were pregnant (Table 7). Other common reasons for not receiving early prenatal care were structural or financial barriers in the health care system, with $38.1 \%$ reporting no appointments available, $28.2 \%$ reporting not having money or insurance to pay for the visit, $27.3 \%$ reporting the doctor/health plan would not start care, and $22.5 \%$ not having a Medicaid card. Reasons women reported not receiving prenatal care as early as they desired differed depending on race, age, WIC participation, education, number of previous births, marital status, and weight status (Table 8). Younger women, women receiving WIC benefits and women who had less education were more likely to report financial barriers to early prenatal care (i.e. not being able to afford care, not having transportation, not yet having a Medicaid card). Women participating in WIC, women who were not married, and women with less than a bachelor's degree were less likely to report health care structural barriers to early prenatal care (i.e. not having appointments available, doctor/health care plan not being willing to start care). 
Table 3 Sociodemographic Characteristics associated with Gestational Age When Certain of Pregnancy and at First Prenatal Visit

\begin{tabular}{|c|c|c|c|}
\hline \multicolumn{2}{|c|}{ Gestational Age when Certain of Pregnancy (weeks) } & \multicolumn{2}{|c|}{ Gestational Age at First Prenatal Visit (week } \\
\hline Weighted Mean (SE) & $\begin{array}{l}\text { Difference of Marginal } \\
\text { Means } \\
(95 \% \mathrm{CI})\end{array}$ & Weighted Mean (SE) & $\begin{array}{l}\text { Difference } \\
\text { of Marginal } \\
\text { Means } \\
(95 \% \mathrm{CI})\end{array}$ \\
\hline
\end{tabular}

\begin{tabular}{|c|c|c|c|c|}
\hline \multicolumn{5}{|l|}{ Race } \\
\hline White (ref) & $5.7(0.1)$ & - & $8.9(0.1)$ & - \\
\hline Black & $7.2(0.3)$ & $1.5(0.9,2.0)$ & $10.1(0.4)$ & $1.2(0.4,2.0)$ \\
\hline Asian & $5.6(0.3)$ & $-0.1(-0.7,0.4)$ & $10.9(0.6)$ & $2.0(0.8,3.2)$ \\
\hline Native North American & $7.0(0.3)$ & $1.3(0.7,1.9)$ & $10.1(0.4)$ & $1.2(0.3,2.1)$ \\
\hline Other race & $6.3(0.3)$ & $0.6(0.0,1.1)$ & $9.5(0.3)$ & $0.5(0.0,1.1)$ \\
\hline Mixed race & $7.0(0.6)$ & $1.3(0.0,2.6)$ & $9.6(0.5)$ & $0.7(-0.3,1.6)$ \\
\hline \multicolumn{5}{|l|}{ Ethnicity } \\
\hline Non-Hispanic (ref) & $5.9(0.1)$ & - & $9.1(0.1)$ & - \\
\hline Hispanic & $6.6(0.2)$ & $0.7(0.3,1.1)$ & $9.6(0.2)$ & $0.5(0.0,0.9)$ \\
\hline \multicolumn{5}{|l|}{ Maternal Age } \\
\hline$<18$ & $10.5(1.0)$ & $4.8(2.8,6.7)$ & $17.6(2.4)$ & $9.1(4.5,13.8)$ \\
\hline $18-24$ & $6.8(0.2)$ & $1.0(0.5,1.6)$ & $10.6(0.3)$ & $2.2(1.6,2.7)$ \\
\hline $25-34$ & $5.7(0.1)$ & $0.0(-0.5,0.4)$ & $8.8(0.1)$ & $0.3(0.0,0.7)$ \\
\hline$\geq 35$ (ref) & $5.8(0.2)$ & - & $8.4(0.2)$ & - \\
\hline \multicolumn{5}{|c|}{ Nutrition program for Women, Infant and Child participation } \\
\hline Yes & $6.6(0.1)$ & $0.9(0.6,1.3)$ & $9.9(0.2)$ & $1.0(0.6,1.4)$ \\
\hline No (ref) & $5.7(0.1)$ & - & $8.8(0.1)$ & - \\
\hline \multicolumn{5}{|l|}{ Education } \\
\hline$<$ High school degree & $7.7(0.4)$ & $2.6(1.9,3.4)$ & $11.9(0.4)$ & $4.1(3.2,4.9)$ \\
\hline High school graduate/GED & $6.7(0.2)$ & $1.7(1.2,2.1)$ & $10.2(0.2)$ & $2.4(1.9,2.8)$ \\
\hline Some college/associate degree & $6.0(0.1)$ & $0.9(0.6,1.3)$ & $8.8(0.1)$ & $1.0(0.7,1.3)$ \\
\hline$\geq$ Bachelor's degree (ref) & $5.0(0.1)$ & - & $7.8(0.1)$ & - \\
\hline \multicolumn{5}{|l|}{ Previous Births } \\
\hline 0 births & $5.8(0.1)$ & $-1.1(-1.7,-0.5)$ & $9.0(0.2)$ & $-1.8(-2.5,-1.2)$ \\
\hline $1-2$ births & $6.0(0.1)$ & $-0.9(-1.6,-0.3)$ & $9.0(0.1)$ & $-1.9(-2.5,-1.2)$ \\
\hline$\geq 3$ births (ref) & $6.9(0.3)$ & - & $10.9(0.3)$ & - \\
\hline \multicolumn{5}{|l|}{ Marital Status } \\
\hline Married (ref) & $5.4(0.1)$ & - & $8.4(0.1)$ & - \\
\hline Not married & $7.1(0.2)$ & $1.7(1.3,2.0)$ & $10.6(0.2)$ & $2.1(1.7,2.6)$ \\
\hline \multicolumn{5}{|l|}{ Weight Status } \\
\hline Underweight & $6.7(0.4)$ & $0.7(-0.1,1.5)$ & $9.8(0.4)$ & $0.7(-0.1,1.4)$ \\
\hline Normal Weight (ref) & $5.9(0.1)$ & - & $9.1(0.1)$ & - \\
\hline Overweight & $5.5(0.1)$ & $-0.4(-0.8,0.0)$ & $9.2(0.2)$ & $0.1(-0.4,0.6)$ \\
\hline Obese & $6.4(0.2)$ & $0.4(-0.1,0.9)$ & $9.3(0.2)$ & $0.2(-0.3,0.7)$ \\
\hline
\end{tabular}

Bold text indicates the difference between the level of interest and the reference is significant with $95 \%$ confidence

\section{Discussion}

While preconception counseling is the gold standard for care to prepare women for a healthy pregnancy by detecting or treating co-morbidities or assisting women with changing health behaviors, $50 \%$ of pregnancies are unplanned (Finer \& Zolna, 2014) and so many women miss this opportune time for counseling. Therefore, optimizing the delivery of prenatal care as early as possible is essential to support the health of women and infants. The majority of the sample (92\%) was able to receive prenatal care in the first trimester, with participants reporting having their first prenatal care appointment around 9 weeks gestation, on average. However, the data demonstrated a two-week gap among women between being certain of their pregnancy and receiving their first prenatal 
Table 4 Multiple Linear Regression Model of Sociodemographic

Characteristics associated with Gestational Age When Certain of Pregnancy and at First Prenatal Visit

\begin{tabular}{|c|c|c|c|c|}
\hline & \multicolumn{2}{|c|}{$\begin{array}{l}\text { Gestational Age when Certain of } \\
\text { Pregnancy (weeks) }\end{array}$} & \multicolumn{2}{|c|}{$\begin{array}{l}\text { Gestational Age at First Prenatal } \\
\text { Visit (weeks) }\end{array}$} \\
\hline & $\begin{array}{l}\text { Estimated Regression } \\
\text { Coefficient }(95 \% \mathrm{CI})\end{array}$ & $\begin{array}{l}\text { Tests of } \\
\text { Model } \\
\text { Effects }^{\mathrm{a}}\end{array}$ & $\begin{array}{l}\text { Estimated Regression } \\
\text { Coefficient }(95 \% \mathrm{CI})\end{array}$ & $\begin{array}{l}\text { Tests of } \\
\text { Model } \\
\text { Effects }^{\mathrm{a}}\end{array}$ \\
\hline \multicolumn{5}{|l|}{ Race } \\
\hline White (ref) & - & 0.010 & - & 0.001 \\
\hline Black & $0.9(0.3,1.5)$ & & $-0.1(-0.8,0.5)$ & \\
\hline Asian & $0.6(0,1.2)$ & & $2.8(1.5,4.0)$ & \\
\hline Native North American & $0.8(0.2,1.4)$ & & $-0.1(-1.2,1.0)$ & \\
\hline Other race & $0.1(-0.7,0.5)$ & & $-0.4(-1.1,0.3)$ & \\
\hline Mixed race & $0.9(-0.4,2.1)$ & & $0.1(-0.9,1.1)$ & \\
\hline \multicolumn{5}{|l|}{ Ethnicity } \\
\hline Non-Hispanic (ref) & - & 0.671 & - & 0.222 \\
\hline Hispanic & $0.1(-0.5,0.7)$ & & $-0.3(-0.9,0.2)$ & \\
\hline \multicolumn{5}{|l|}{ Maternal Age } \\
\hline$<18$ & 3.6(1.6, 5.5) & 0.001 & $7.8(2.9,12.8)$ & $<0.001$ \\
\hline $18-24$ & $0.3(-0.3,1.0)$ & & $1.7(1.1,2.3)$ & \\
\hline $25-34$ & $-0.1(-0.6,0.4)$ & & $0.5(0.1,0.8)$ & \\
\hline$>35$ (ref) & - & & - & \\
\hline \multicolumn{5}{|c|}{ Nutrition Program for Women, Infant and Child Participation } \\
\hline Yes & $-0.4(-1.0,0.2)$ & 0.151 & $-0.8(-1.3,-0.3)$ & 0.003 \\
\hline No (ref) & - & & - & \\
\hline \multicolumn{5}{|l|}{ Education } \\
\hline$<$ High school degree & $1.9(0.8,3.0)$ & $<0.001$ & $2.9(2.0,3.9)$ & $<0.001$ \\
\hline High school graduate/GED & $1.2(0.5,2.0)$ & & $1.8(1.2,2.3)$ & \\
\hline Some college/associate degree & $0.6(0.2,1.0)$ & & $0.7(0.3,1.1)$ & \\
\hline$\geq$ Bachelor's degree (ref) & - & & - & \\
\hline \multicolumn{5}{|l|}{ Previous Births } \\
\hline 0 births & $-1.0(-1.6,-0.4)$ & 0.002 & $-2.0(-2.6,-1.4)$ & $<0.001$ \\
\hline $1-2$ births & $-0.6(-1.2,0.1)$ & & $-1.6(-2.2,-1.0)$ & \\
\hline$\geq 3$ births (ref) & - & & - & \\
\hline \multicolumn{5}{|l|}{ Marital Status } \\
\hline Married (ref) & - & 0.001 & - & $<0.001$ \\
\hline Not married & $0.8(0.3,1.3)$ & & $1.2(0.8,1.7)$ & \\
\hline \multicolumn{5}{|l|}{ Weight Status } \\
\hline Underweight & $0.2(-0.5,1.0)$ & 0.002 & $-0.5(-1.4,0.4)$ & 0.573 \\
\hline Normal Weight (ref) & - & & - & \\
\hline Overweight & $-0.6(-1.0,-0.2)$ & & $-0.1(-0.6,0.3)$ & \\
\hline Obese & $0.1(-0.5,0.6)$ & & $0.1(-0.4,0.6)$ & \\
\hline
\end{tabular}

Bold text indicates the difference between the level of interest and the reference is significant with $95 \%$ confidence

${ }^{\text {ap }} \mathrm{P}$ value based on F-test for Type 3 Analysis of Effects care appointment, with a larger gap among the $15 \%$ of women (almost 600,000 women (Centers for Disease Control \& Prevention, 2017) in the United States each year) who reported not receiving prenatal care as early as they wished. Significant systemic and socioeconomic barriers to initial prenatal care visits were identified such as no available appointments, not having money/insurance to pay for the visit, doctor/health plan being unwilling to start care until later in pregnancy, and not having a Medicaid card.

The data also showed clear health disparities in how early prenatal care is received by vulnerable populations of women who are at highest risk for pregnancy-related mortality (Creanga et al., 2017). Specifically, women who 
Table 5 Socio-demographic Characteristics by Receiving Prenatal Care as Early as would have Liked or Not

\begin{tabular}{|c|c|c|}
\hline Characteristic & $\begin{array}{l}\text { Received Prenatal Care as Early as } \\
\text { would have Like } \\
\mathrm{N}^{\mathrm{a}}(\%)^{\mathrm{b}}\end{array}$ & $\begin{array}{l}\text { Did not Receive Prenatal Care } \\
\text { as Early as would have Liked } \\
\mathrm{N}(\%)\end{array}$ \\
\hline \multicolumn{3}{|l|}{ Race } \\
\hline White & $7549(61.7 \%)$ & $1156(10.4 \%)$ \\
\hline Black & $2450(11.6 \%)$ & $540(2.4 \%)$ \\
\hline $\begin{array}{l}\text { Asian (Chinese, Japanese, Filipino, other } \\
\text { Asian) }\end{array}$ & $691(4.4 \%)$ & $106(0.6 \%)$ \\
\hline Native North American (American Indian, Hawaiian, Alaskan native) & $389(0.6 \%)$ & $133(0.2 \%)$ \\
\hline Other races & $421(4.4 \%)$ & $104(1.3 \%)$ \\
\hline Mixed race & $487(2.0 \%)$ & $100(0.3 \%)$ \\
\hline \multicolumn{3}{|l|}{ Ethnicity } \\
\hline Hispanic & $1955(20.9 \%)$ & $412(5.0 \%)$ \\
\hline Non-Hispanic & $10,150(63.9 \%)$ & $1740(10.2 \%)$ \\
\hline \multicolumn{3}{|l|}{ Age (years) } \\
\hline$<18$ & $170(1.1 \%)$ & $63(0.5 \%)$ \\
\hline $18-24$ & $2728(18.4 \%)$ & $695(5.3 \%)$ \\
\hline $25-34$ & $7186(51.2 \%)$ & $1132(7.6 \%)$ \\
\hline$\geq 35$ & $2080(14.0 \%)$ & $276(1.8 \%)$ \\
\hline \multicolumn{3}{|l|}{ Nutrition program for Women, Infant and Child participation } \\
\hline Yes & $4525(31.5 \%)$ & $1027(7.1 \%)$ \\
\hline No & $7459(53.3 \%)$ & $1106(8.1 \%)$ \\
\hline \multicolumn{3}{|l|}{ Maternal years of education } \\
\hline Less than a high school degree & $1586(11.4 \%)$ & $378(2.5 \%)$ \\
\hline High school graduate/GED & $2941(20.5 \%)$ & $692(5.2 \%)$ \\
\hline Some college/Associate degree & $3364(22.2 \%)$ & $684(4.3 \%)$ \\
\hline Bachelor's degree or higher & $4184(30.7 \%)$ & $398(3.2 \%)$ \\
\hline \multicolumn{3}{|l|}{ Number of previous live births } \\
\hline 0 & $4614(30.8 \%)$ & $889(6.5 \%)$ \\
\hline $1-2$ & $6008(44.5 \%)$ & $930(6.7 \%)$ \\
\hline$\geq 3$ & $1525(9.5 \%)$ & $342(1.9 \%)$ \\
\hline \multicolumn{3}{|l|}{ Married } \\
\hline Yes & $7204(52.9 \%)$ & $886(7.3 \%)$ \\
\hline No & $4949(31.9 \%)$ & $1278(7.9 \%)$ \\
\hline \multicolumn{3}{|l|}{ BMI category } \\
\hline \multicolumn{3}{|l|}{ Underweight $(<18.5)$} \\
\hline Normal (18.5-24.9) & $395(2.7 \%)$ & $92(0.7 \%)$ \\
\hline Overweight (25-29.9) & $5316(39.8 \%)$ & $936(7.0 \%)$ \\
\hline \multirow[t]{2}{*}{ Obese $(\geq 30)$} & $2916(22.1 \%)$ & $485(3.4 \%)$ \\
\hline & $3049(20.4 \%)$ & $558(3.9 \%)$ \\
\hline
\end{tabular}

a Number of women from unweighted sample distribution

${ }^{\mathrm{b}}$ Percentages were weighted to account for deliberate survey oversampling and nonresponse and non-coverage

identified as Black and Native American learned of pregnancy significantly later and established prenatal care later compared to women who identified as white. Given the higher burden of adverse outcomes among Black and Native American women (Heck et al., 2021; Kuehn, 2019; Kuriya et al., 2016; Singh, 2021), changes in policy (e.g. recommendations for a specific week to start prenatal care from professional organization) and insurance coverage will be necessary to prioritize early prenatal care in these populations. Women who were 24 years or younger, women who were not married, women with lower educational attainment, and women who were receiving WIC benefits similarly confirmed their pregnancies later and also received prenatal care later. Given the most common barrier to timely prenatal 
Table 6 Sociodemographic Characteristics associated with not Receiving Prenatal Care as Early as Would have Liked

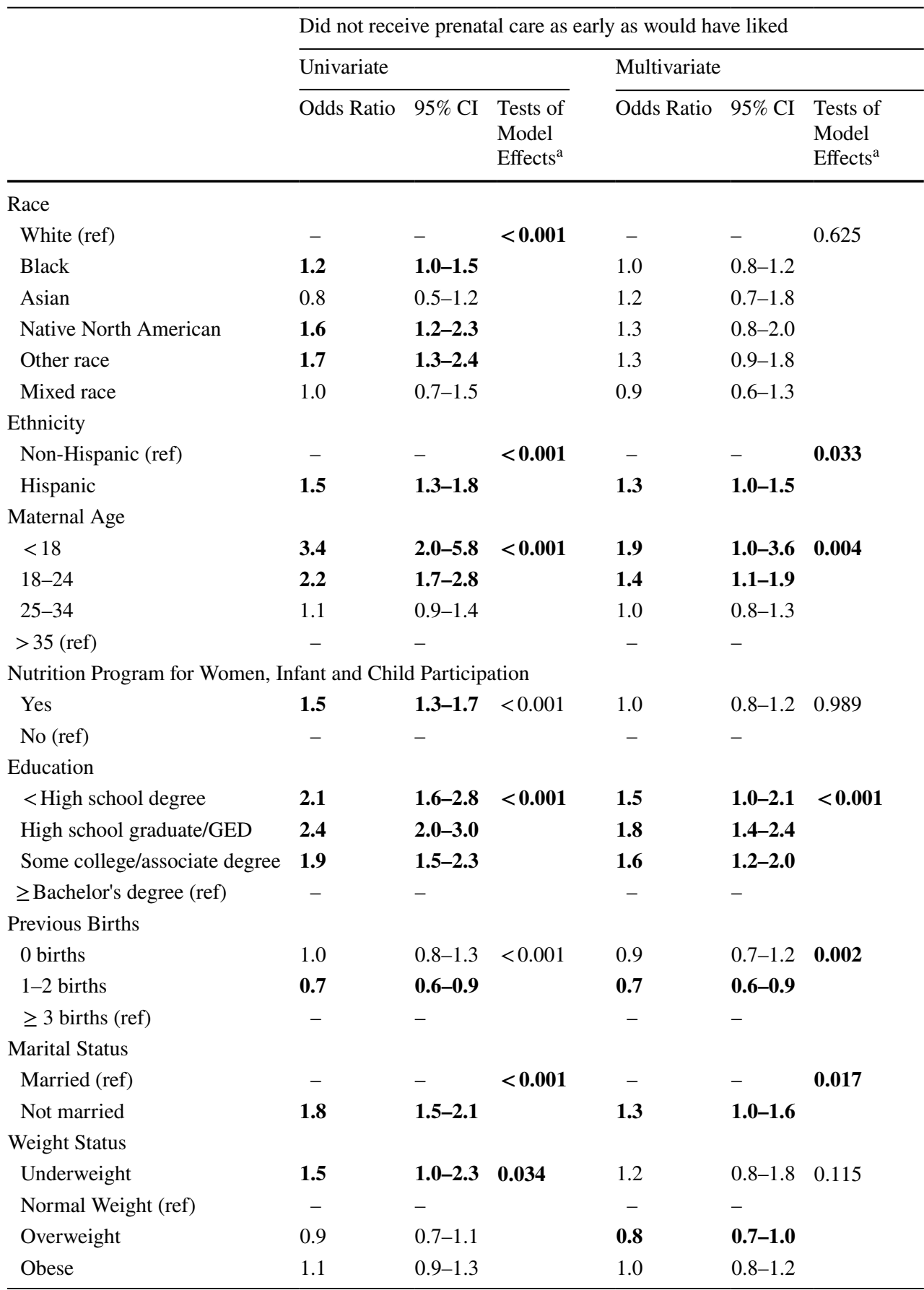

Bold text indicates the difference between the level of interest and the reference is significant with $95 \%$ confidence

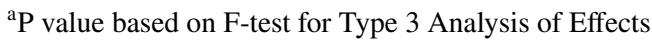

care was lack of early identification of pregnancy, it may be helpful to enhance efforts reducing rates of unintended pregnancies through facilitating birth control access (Kallner \& Danielsson, 2016). In addition, increasing awareness of the importance of receiving prenatal care early may encourage women of childbearing age to act quickly to access care when they suspect they are pregnant. However, early recognition of pregnancy may not eliminate the issue of accessing early prenatal care, since women in disadvantaged populations reported that lack of appointments and reluctance of providers to provide early prenatal appointments were key barriers to receiving early prenatal care.

Women who were participating in WIC (a proxy for lower income) and had less educational attainment were 
Table 7 Perceived barriers to receiving early prenatal care

\begin{tabular}{ll}
\hline Perceived barrier & $\mathrm{N}^{\mathrm{a}}(\%)^{\mathrm{b}}$ \\
\hline Was not aware of pregnancy status & $917(42.3 \%)$ \\
No appointments available when they wanted one & $755(38.1 \%)$ \\
Unable to afford the visits & $452(28.2 \%)$ \\
Doctor or health plan would not start prenatal care as & $463(27.3 \%)$ \\
$\quad$ early as they wanted & $392(22.5 \%)$ \\
No Medicaid card & $326(15.4 \%)$ \\
Too busy & $238(10.7 \%)$ \\
Lack of transportation & $221(9.5 \%)$ \\
Did not want to disclose pregnancy status & $184(8.2 \%)$ \\
No leave at work/school & $134(7.0 \%)$ \\
Lack of child care did not want prenatal care & $92(3.2 \%)$ \\
\hline
\end{tabular}

${ }^{a}$ Number of women from unweighted sample distribution

b Percentages were weighted to account for deliberate survey oversampling and nonresponse and non-coverage

more likely to report both financial barriers (i.e. inability to afford medical care, not having transportation, not yet having her Medicaid card) and systemic barriers (i.e. doctor/health plan not offering early care or appointment availability) compared to women with higher incomes and more education. Understanding and intervening on these barriers is crucial in facilitating prenatal care as early as possible in pregnancy, in order to reduce morbidity and mortality for both maternal and fetal health (Bingham et al., 2011; Maternal Health Task Force). Potential considerations to facilitate early prenatal care include: 1) facilitating early prenatal care appointments among Medicaid-eligible women prior to receiving their card (Palmer, 2020; Piper et al., 1994), 2) increasing awareness among pregnant women that a variety of providers can offer the initial prenatal care visit (including family physicians) (Kozhimannil et al., 2012), and 3) supporting technology-based prenatal care visits (Marko et al., 2019; Peahl et al., 2020). In addition, dividing the global obstetrics fee into components, including an "initial prenatal care visit" component, could provide appropriate incentives for clinics to provide early prenatal care, even if there is a first trimester pregnancy loss (Applegate et al., 2014). Further, onsite childcare or child-friendly clinic spaces could address the challenge of lack of child care during these medical appointments (Phillippi, 2009). Lastly, ensuring the scheduling process is simple for those with the lowest levels of literacy or of experience with the medical community may remove barriers for young women and those with low levels of education.

Consistent with previous research (Lynch et al., 2014), it is notable that no disparities were detected in gestational age at the first prenatal care visit by pre-gravid BMI category, despite the prevalence of weight stigma in health care settings and health care avoidance among individuals with overweight or obesity (Mensinger et al., 2018). Despite the greater likelihood of excessive gestational weight gain (Deputy et al., 2015) and the increased prevalence of maternal health complications among women with overweight and obesity (Wei et al., 2016), we found no differences in the timing of prenatal care based on BMI category. Nonetheless, since gestational weight gain in the first trimester appears to have a disproportionate impact on the development of gestational diabetes (MacDonald et al., 2017), it may be beneficial for all women to receive prenatal care with clear recommendations for gestational weight gain earlier in the first trimester.

This study has several key strengths and limitations. First, our research has the strength of using a large, national dataset, allowing for an adequate sample size for examining the effects of several sociodemographic variables. However, due to the large sample size, some differences detected may be statistically significant but not clinically meaningful. While we utilized a national data set, which recruited a representative sample within each state, not all states administered every question that we utilized in these analyses and one question (i.e. gestational age at pregnancy confirmation) was only administered by four states; thus the degree to which the results are generalizable is unknown. Furthermore, women who respond to the PRAMS survey may differ from the general population (e.g. characteristics like conscientiousness and altruism). In addition, data utilized in these analyses were based on maternal self-report and are therefore subject to the typical limitations of self-reported data (e.g. ability to recall accurate information about their prenatal care, providing social acceptable rather than entirely accurate responses). Thus, delayed prenatal care and barriers may be even more common than was reported in this article. Finally, the COVID-19 pandemic has led to many dramatic changes in the provision of health care (e.g. telehealth), and future research should examine whether the timing of the initial prenatal care visit or the prevalence of barriers has changed.

In summary, this study illustrates a window of opportunity to provide earlier prenatal care. Early prenatal care is preferred by many women and would facilitate important medical and behavioral treatments critical for maternal and fetal health to be addressed as early as possible. This study also identified common barriers to care, overall and among specific vulnerable population groups. Strategies to address these barriers on the patient, provider and systemic levels is warranted. 


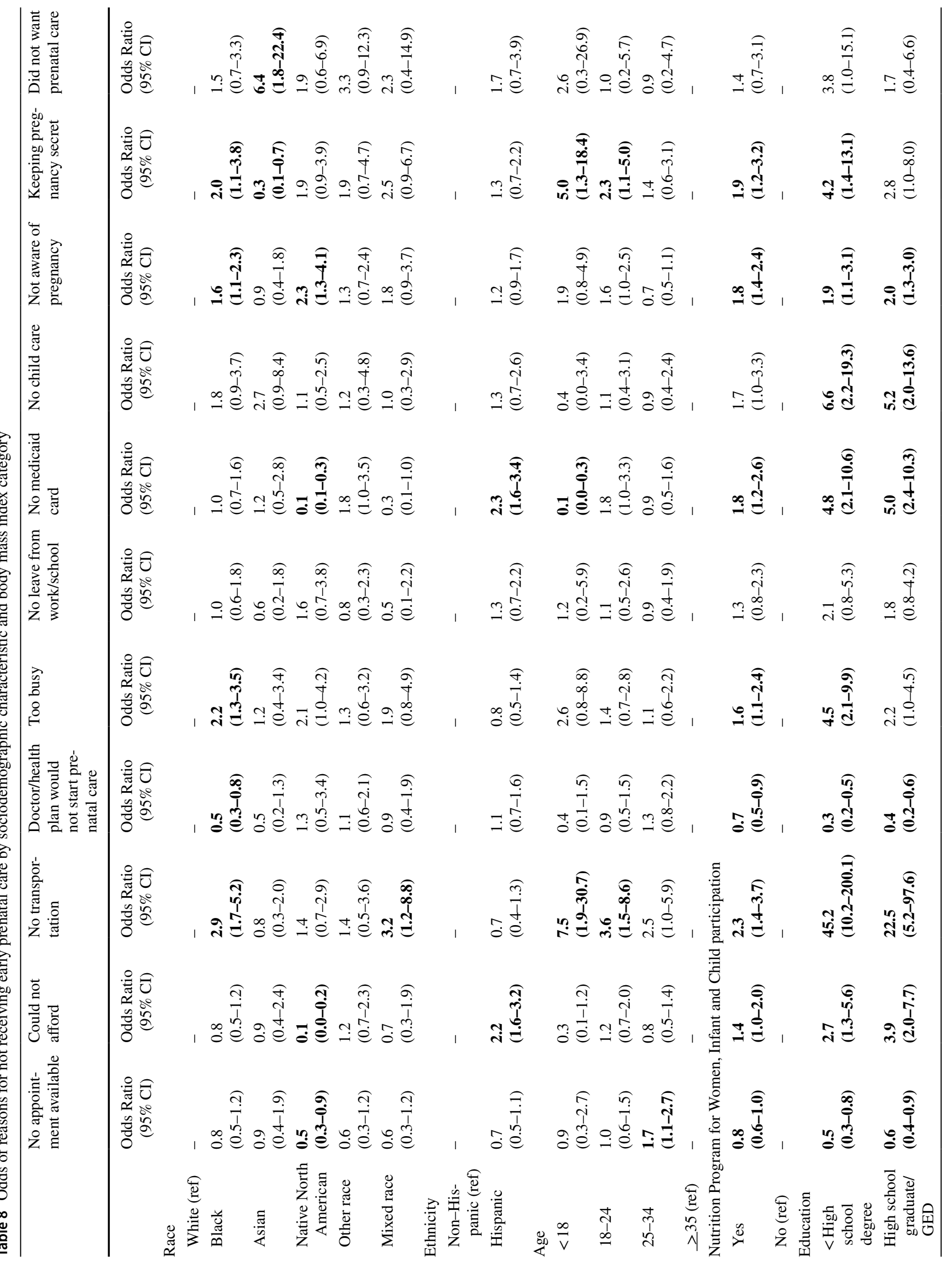




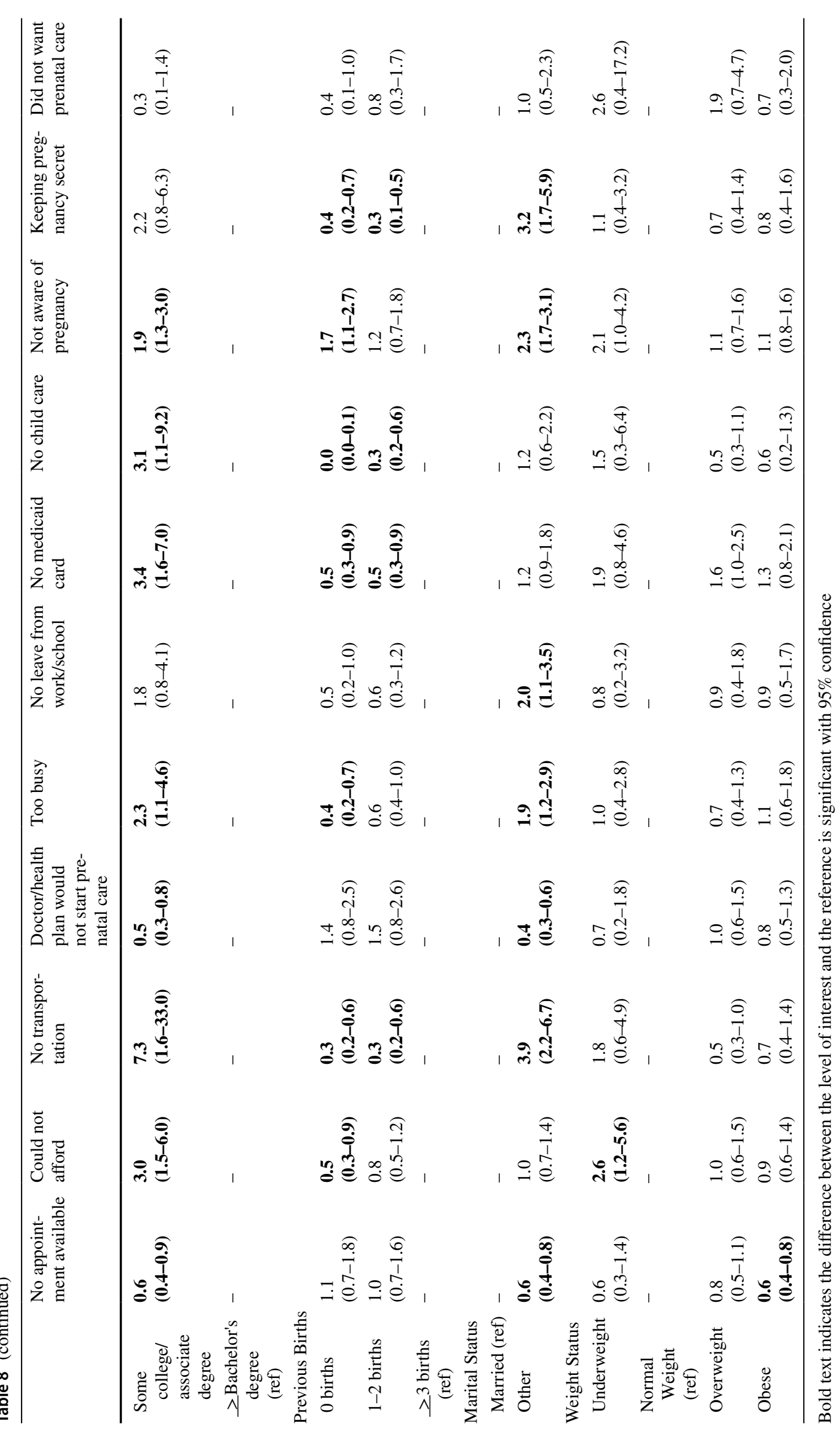


Acknowledgements We would like to acknowledge the Pregnancy Risk Assessment Monitoring System (PRAMS) working group and the Centers for Disease Control and Prevention for providing access to the PRAMS dataset for this analysis. We would also like to acknowledge Christina M. Frank, University of Kansas, School of Medicine-Wichita, for her work on in-text citations and references.

Authors' contributions All authors contributed to study design, drafting of the manuscript, and review of the final manuscript. C.Y.C. \& J. W. conducted the data analyses.

Funding Not applicable.

Data availability The data are available from Pregnancy Risk Assessment Monitoring System (PRAMS) working group and the Centers for Disease Control and Prevention.

Code availability Not applicable.

\section{Declarations}

Conflict of interest The authors declare that they have no conflict of interest.

Ethical Approval The study was determined to be exempt from University of Tennessee Health Science Center Institutional Review Board approval because no identifiable private information was utilized and have therefore been performed in accordance with the ethical standards laid down in the 1964 Declaration of Helsinki and its later amendments;

Consent to Participate All participants received a written informed consent statement.

Consent for Publication Not applicable.

\section{References}

American Academy of Pediatrics Committee on Fetus and Newborn. (2012). Guidelines for Perinatal Care (7th edition ed.).

Albrecht, S. S., Kuklina, E. V., Bansil, P., Jamieson, D. J., Whiteman, M. K., Kourtis, A. P., Posner, S. F., \& Callaghan, W. M. (2010). Diabetes trends among delivery hospitalizations in the US, 1994-2004. Diabetes Care, 33(4), 768-773.

Applegate, M., Gee, R. E., \& Martin, J. N. (2014). Improving maternal and infant health outcomes in Medicaid and the children's health insurance program. Obstetrics \& Gynecology, 124(1), 143-149.

Bingham, D., Strauss, N., \& Coeytaux, F. (2011). Maternal mortality in the United States: A human rights failure. Contraception, 83(3), 189-193.

Centers for Disease Control and Prevention. (2017). National Center for Health Statistics. Retrieved November 4, 2019 from https:// www.cdc.gov/nchs/fastats/births.htm.

Centers for Disease Control and Prevention. (2019b). Reproductive Health. Retrieved December 1, 2019 from https://www.cdc.gov/ reproductivehealth/maternalinfanthealth/pregnancy-mortalitysurveillance-system.htm.

Centers for Disease Control and Prevention. (2019a). Pregnancy Risk Assessment Monitoring Survey. Retrieved October 22, 2019 from https://www.cdc.gov/prams/.
Creanga, A. A., Syverson, C., Seed, K., \& Callaghan, W. M. (2017). Pregnancy-related mortality in the United States, 2011-2013. Obstetrics and Gynecology, 130(2), 366.

Deputy, N. P., Sharma, A. J., \& Kim, S. Y. (2015). Gestational weight gain-United States, 2012 and 2013. MMWR. Morbidity and Mortality Weekly Report, 64(43), 1215.

Finer, L. B., \& Zolna, M. R. (2014). Shifts in intended and unintended pregnancies in the United States, 2001-2008. American Journal of Public Health, 104(S1), S43-S48.

Goldstein, R. F., Abell, S. K., Ranasinha, S., Misso, M., Boyle, J. A., Black, M. H., Li, N., Hu, G., Corrado, F., \& Rode, L. (2017). Association of gestational weight gain with maternal and infant outcomes: A systematic review and meta-analysis. Journal of the American Medical Association, 317(21), 2207-2225.

Heck, J. L., Jones, E. J., Bohn, D., McCage, S., Parker, J. G., Parker, M., Pierce, S. L., \& Campbell, J. (2021). Maternal mortality among American Indian/Alaska Native women: A scoping review. Journal of Women's Health, 30(2), 220-229.

Kallner, H. K., \& Danielsson, K. G. (2016). Prevention of unintended pregnancy and use of contraception-important factors for preconception care. Upsala Journal of Medical Sciences, 121(4), 252-255.

Kozhimannil, K. B., Avery, M. D., \& Terrell, C. A. (2012). Recent trends in clinicians providing care to pregnant women in the United States. Journal of Midwifery \& Women's Health, 57(5), 433-438.

Kuehn, B. (2019). Disparities in maternal mortality. Journal of the American Medical Association, 322(16), 1545-1545.

Kuklina, E. V., \& Callaghan, W. M. (2011). Chronic heart disease and severe obstetric morbidity among hospitalisations for pregnancy in the USA: 1995-2006. BJOG: an International Journal of Obstetrics \& Gynaecology, 118(3), 345-352.

Kuklina, E. V., Ayala, C., \& Callaghan, W. M. (2009). Hypertensive disorders and severe obstetric morbidity in the United States. Obstetrics \& Gynecology, 113(6), 1299-1306.

Kuriya, A., Piedimonte, S., Spence, A. R., Czuzoj-Shulman, N., Kezouh, A., \& Abenhaim, H. A. (2016). Incidence and causes of maternal mortality in the USA. Journal of Obstetrics and Gynaecology Research, 42(6), 661-668.

Lynch, C. D., Tumin, R., \& Prasad, M. R. (2014). Association between body mass index and the timing of pregnancy recognition and entry into prenatal care. Obstetrics \& Gynecology, 124(5), 911-918.

MacDonald, S. C., Bodnar, L. M., Himes, K. P., \& Hutcheon, J. A. (2017). Patterns of gestational weight gain in early pregnancy and risk of gestational diabetes mellitus. Epidemiology (cambridge, Mass.), 28(3), 419.

Marko, K. I., Ganju, N., Krapf, J. M., Gaba, N. D., Brown, J. A., Benham, J. J., Oh, J., Richards, L. M., \& Meltzer, A. C. (2019). A mobile prenatal care app to reduce in-person visits: prospective controlled trial. JMIR mHealth and uHealth, 7(5), e10520.

Maternal Health Task Force. Maternal Health in the United States. Retrieved November 4, 2019 from https://www.mhtf.org/topics/ maternal-health-in-the-united-states/.

Mensinger, J. L., Tylka, T. L., \& Calamari, M. E. (2018). Mechanisms underlying weight status and healthcare avoidance in women: A study of weight stigma, body-related shame and guilt, and healthcare stress. Body Image, 25, 139-147.

Michelle, J., \& Osterman, M. (2016). Timing and adequacy of prenatal care in the United States. National Vital Statistics Report, 67, 1-14.

Moos, M.-K., Dunlop, A. L., Jack, B. W., Nelson, L., Coonrod, D. V., Long, R., Boggess, K., \& Gardiner, P. M. (2008). Healthier women, healthier reproductive outcomes: Recommendations for the routine care of all women of reproductive age. American Journal of Obstetrics and Gynecology, 199(6), S280-S289. 
Palmer, M. (2020). Preconception subsidized insurance: Prenatal care and birth outcomes by race/ethnicity. Health Economics, 29(9), 1013-1030.

Peahl, A. F., Smith, R. D., \& Moniz, M. H. (2020). Prenatal care redesign: creating flexible maternity care models through virtual care. American Journal of Obstetrics and Gynecology, 223(3), 389.

Phillippi, J. C. (2009). Women's perceptions of access to prenatal care in the United States: A literature review. Journal of Midwifery \& Women's Health, 54(3), 219-225.

Piper, J. M., Mitchel, E. F., Jr., \& Ray, W. (1994). Presumptive eligibility for pregnant Medicaid enrollees: Its effects on prenatal care and perinatal outcome. American Journal of Public Health, 84(10), 1626-1630.

Shulman, H. B., Gilbert, B. C., \& Lansky, A. (2006). The Pregnancy Risk Assessment Monitoring System (PRAMS): Current methods and evaluation of 2001 response rates. Public Health Reports, 121(1), 74-83.

Singh, G. K. (2021). Trends and social inequalities in maternal mortality in the United States, 1969-2018. International Journal of Maternal and Child Health and AIDS, 10(1), 29.
Stulberg, D. B., Cain, L., HashamDahlquist, I., \& Lauderdale, D. S. (2017). Pre-pregnancy and Early Prenatal Care are Associated with Lower Risk of Ectopic Pregnancy Complications in the Medicaid Population: 2004-08. Paediatric and Perinatal Epidemiology, 31(1), 4-10.

Wei, Y.-M., Yang, H.-X., Zhu, W.-W., Liu, X.-Y., Meng, W.-Y., Wang, Y.-Q., Shang, L.-X., Cai, Z.-Y., Ji, L.-P., \& Wang, Y.-F. (2016). Risk of adverse pregnancy outcomes stratified for pre-pregnancy body mass index. The Journal of Maternal-Fetal \& Neonatal Medicine, 29(13), 2205-2209.

Yan, J. (2017). The effects of prenatal care utilization on maternal health and health behaviors. Health Economics, 26(8), 1001-1018.

Publisher's Note Springer Nature remains neutral with regard to jurisdictional claims in published maps and institutional affiliations. 